This is an electronic reprint of the original article. This reprint may differ from the original in pagination and typographic detail.

Author(s): Nordbo, Annika; Kekäläinen, Pekka; Siivola, Erkki; Lehto, Roope; Vesala, Timo; Timonen, Jussi

Title: $\quad$ Tube transport of water vapor with condensation and desorption

Year: $\quad 2013$

Version:

Please cite the original version:

Nordbo, A., Kekäläinen, P., Siivola, E., Lehto, R., Vesala, T., \& Timonen, J. (2013). Tube transport of water vapor with condensation and desorption. Applied Physics Letters, 102, Article 194101. https://doi.org/10.1063/1.4804639

All material supplied via JYX is protected by copyright and other intellectual property rights, and duplication or sale of all or part of any of the repository collections is not permitted, except that material may be duplicated by you for your research use or educational purposes in electronic or print form. You must obtain permission for any other use. Electronic or print copies may not be offered, whether for sale or otherwise to anyone who is not an authorised user. 


\section{AlP |Applied Physics \\ Letters}

\section{Tube transport of water vapor with condensation and desorption}

Annika Nordbo, Pekka Kekäläinen, Erkki Siivola, Roope Lehto, Timo Vesala, and Jussi Timonen

Citation: Applied Physics Letters 102, 194101 (2013); doi: 10.1063/1.4804639

View online: http://dx.doi.org/10.1063/1.4804639

View Table of Contents: http://scitation.aip.org/content/aip/journal/apl/102/19?ver=pdfcov

Published by the AIP Publishing

\section{Articles you may be interested in}

Marangoni condensation of ammonia-water vapor mixtures on a horizontal tube

AIP Conf. Proc. 1547, 373 (2013); 10.1063/1.4816887

Mechanisms of high quality $\mathrm{i}-\mathrm{ZnO}$ thin films deposition at low temperature by vapor cooling condensation technique

J. Appl. Phys. 108, 073119 (2010); 10.1063/1.3477325

On the capillary condensation of water in mesoporous silica films measured by $x$-ray reflectivity

Appl. Phys. Lett. 87, 223105 (2005); 10.1063/1.2136412

Capillary condensation monitored in birefringent porous silicon layers

J. Appl. Phys. 94, 4913 (2003); 10.1063/1.1609643

Capillary condensation of nitrogen in MCM-41 and SBA-15

J. Chem. Phys. 117, 8036 (2002); 10.1063/1.1510440

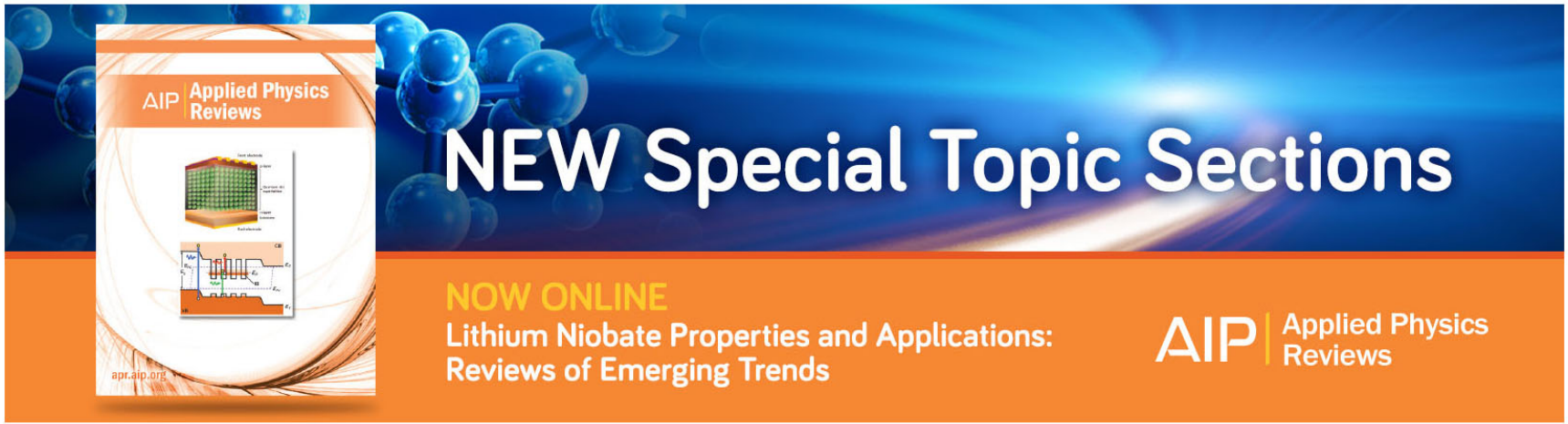




\title{
Tube transport of water vapor with condensation and desorption
}

\author{
Annika Nordbo, ${ }^{1, a)}$ Pekka Kekäläinen, ${ }^{2}$ Erkki Siivola, ${ }^{3}$ Roope Lehto, ${ }^{2}$ Timo Vesala, ${ }^{1}$ \\ and Jussi Timonen 2,4 \\ ${ }^{1}$ Department of Physics, University of Helsinki, P.O. Box 48, 00014, Helsinki, Finland \\ ${ }^{2}$ Department of Physics, University of Jyväskylä, P.O. Box 35, 40014, Jyväskylä, Finland \\ ${ }^{3}$ Department of Physics, University of Helsinki, P.O. Box 64, 00014, Helsinki, Finland \\ ${ }^{4}$ NanoScience Center, University of Jyväskylä, P.O. Box 35, 40014, Jyväskylä, Finland
}

(Received 29 January 2013; accepted 25 April 2013; published online 13 May 2013)

\begin{abstract}
Attenuation and delay of active tracers in tube transport is an important current problem, but its full explanation is still lacking. To this end a model is introduced, where part of a tracer undergoes condensation and evaporation, treated as a diffusion-type process, in addition to Taylor dispersion. Condensation of water was verified by high-speed imaging, and the model solution fitted the breakthrough curves of laboratory measurements with pulses of water vapor of varying relative humidity. The model provides a transfer function whose performance was verified against field measurements. @ 2013 AIP Publishing LLC. [http://dx.doi.org/10.1063/1.4804639]
\end{abstract}

Sampling of air through a tube so as to measure the concentration of tracers it may contain is an old, but even now a common measuring technique used in numerous applications. ${ }^{1-5}$ Unfortunately, the resolution of such measurements is often degraded by dispersion effects which can both attenuate and delay important fluctuations. Such effects increase with tube length. In the case the tracer is a passive scalar, dispersion by differential advection caused by the flow profile, e.g., via Taylor dispersion, and by molecular and turbulent diffusion, has traditionally been suggested as the responsible mechanisms. ${ }^{6-11}$ In the case of a sorbing ("active") tracer like water vapor, additional dispersion, and delay can be expected to be caused by adsorption and desorption processes ("sorption") at the tube wall, as attenuation is so strong that it cannot be explained by passive-tracer models. ${ }^{11,12}$ In spite of this expectation, the (old) problem of tube transport of an active tracer has not been properly solved, although attenuation in, e.g., water-vapor fluctuations can cause for instance a 30\% underestimation in measurements of evaporation from a ground surface to the atmosphere. ${ }^{5}$ A semi-empirical treatment of sorption effects of water vapor has been tried in order to predict attenuation, but it suffered from parametrization problems ${ }^{13}$ and did not lead to further work in this direction. Because of its importance, the problem has, however, received much attention during the past ten years. ${ }^{3-5}$ Recent work has mostly focused on empirically correcting for the missing variation in data. ${ }^{4,14}$ It is evident that a fresh look at the effect of sorption processes is called for so as to be able to correct for dispersion effects in active-scalar measurements.

We thus introduce a model for the sorption processes that appear in a tube flow of an active tracer (here water). For a pulse of water vapor, the main assumption is that drops of water begin to grow at the tube wall when the pulse is introduced, a dynamic equilibrium in drop size is reached, and evaporation begins to dominate at the trailing phase of the pulse leading eventually to vanishing of the drops. This phenomenon is expected to explain the attenuation as well as

\footnotetext{
${ }^{a)}$ Email: annika.nordbo@helsinki.fi
}

the delay of the pulse in a tube flow. The model was solved for the breakthrough curve (the pulse at the end of the tube) of the tracer, and it was tested using laboratory measurements on pulses of water vapor advected along a tube. A transfer function was derived based on the model, and it was applied to field-measurement data that represented a continuous sampling of air of varying humidity. The drop formation mechanism was tested by imaging a transparent section of the tube.

At a molecular level, a tracer molecule absorbed in a sessile drop undergoes Brownian motion until it is near the surface and receives enough energy from thermal fluctuations to escape. Consequent transfer of evaporated molecules across the stagnant boundary layer at the tube wall back to the flow is also diffusive. ${ }^{16,17}$ The whole process of evaporation back to the flow is thus diffusion limited, ${ }^{15}$ and it can be considered as a single diffusion process in which the time spent in the condensed phase is considered as a time delay described by a decreased diffusion coefficient (from that in the boundary layer). We do not know this "effective" diffusion coefficient without solving the whole complicated process. Here this diffusion coefficient is included in parameters ( $\lambda$ and $\kappa$, see below) used to fit measured data with the model, so its value needs not be known a priori. The essential properties of this effective diffusion process can be captured by one-dimensional (1D) diffusion: Advection with molecular diffusion in the tube (in the $x$ direction) is thus coupled to 1D diffusion away from the tube flow, to which we attach an auxiliary ("effective") dimension ( $z$ ) and a length scale $(\ell)$ as the diffusion process is spatially constrained (to the drop and the boundary layer at the tube wall). Note that similar advection-diffusion problems are rather common in transport in porous media. ${ }^{18}$ If the carrier fluid has a tracer concentration $C_{f}(x, t)$ (average over tube cross section at $x$ ) in a control volume $\pi R^{2} d x$, and the part of the concentration condensed at the tube wall in that volume is $C_{m}(x, z, t)$, we have a coupled advection-diffusion equation for the evolution of tracer concentration, given that there is a flow velocity $v$ in a tube of radius $R$ and length $L$, which can be expressed in the form, 


$$
\begin{aligned}
& \frac{\partial C_{f}}{\partial t}+v \frac{\partial C_{f}}{\partial x}-D_{f} \frac{\partial^{2} C_{f}}{\partial x^{2}}=\left.\frac{2 \phi D}{R} \frac{\partial C_{m}}{\partial z}\right|_{z=0} \\
& \frac{\partial C_{m}}{\partial t}-D \frac{\partial^{2} C_{m}}{\partial z^{2}}=0 .
\end{aligned}
$$

Here $D_{f}$ is related to the molecular diffusion coefficient of the tracer (water) in the carrier fluid (air), but it includes the effect of Taylor dispersion in turbulent flow (for more details, see supplemental material ${ }^{19}$ and Ref. 10), $D$ is the effective diffusion coefficient of the tracer in the evaporation process (including diffusion in the liquid phase and across the gaseous boundary layer), and $\phi$ is the fraction of the surface area, where nucleation of drops can take place. The appropriate boundary conditions to be imposed in this case, assuming a delta-function pulse of the tracer (of mass $M_{0}$ ) is introduced into the system, are given by $C_{m}(x, 0, t)=C_{f}(x, t)$, $\frac{\partial C_{m}}{\partial z}(x, \ell, t)=0$, and $C_{f}(0, t)=\frac{M_{0}}{\pi R^{2} v} \delta(t)$, while the initial conditions are given by $C_{m}(x, z, 0)=0$ and $C_{f}(x, 0)=0$. Coupled equations of this kind typically appear in problems like matrix diffusion. ${ }^{20,21}$ The diffusion part of the above equations can be solved independently, and we arrive in an integro-differential equation for $C_{f}$. In dimensionless form, when $\xi=x / L$ is the distance from tube inlet and $\tau=t v / L$ is the time after introduction of the pulse, this equation is given by $^{20}$

$$
\begin{gathered}
\frac{\partial C}{\partial \tau}(\xi, \tau)+\frac{\partial C}{\partial \xi}(\xi, \tau)-\mu^{2} \frac{\partial^{2} C}{\partial \xi^{2}}(\xi, \tau) \\
=-\lambda \int_{0}^{\tau} \Lambda(\tau-\sigma) \frac{\partial C}{\partial \sigma} d \sigma, \\
\Lambda(\tau)=\frac{2}{\kappa} \sum_{n=0}^{\infty} e^{-\left(\gamma_{n}^{2} / \kappa^{2}\right) \tau}, \quad \gamma_{n}=\left(n+\frac{1}{2}\right) \pi .
\end{gathered}
$$

There are thus three dimensionless variables that characterize the system: $\mu=\sqrt{\tilde{D}_{f}}$, related to molecular diffusion in air and to Taylor dispersion caused by turbulent flow, $\lambda=2 \phi L \sqrt{\tilde{D}} / R$, related to the relative area, where drops can form, and $\kappa=\ell /(L \sqrt{\tilde{D}})$, related to the length scale attached to the diffusive evaporation process. Here, we have introduced the dimensionless diffusion coefficients $\tilde{D} \equiv \frac{D}{L v}$ and $\tilde{D}_{f} \equiv \frac{D_{f}}{L v}$.

Equation (2) can best be solved in the form of a series expansion using Laplace transformation, but this solution is complicated and it is only given in supplemental material. ${ }^{19}$ The breakthrough curve, $C_{f}(L, t)$, is found as a convolution of the actual input curve of the tracer and this solution. Moreover, a transfer function $(T F)$ that describes the effect of sorption processes in frequency space, can be derived for the model (by analytic continuation of the Laplace transform, see supplemental material ${ }^{19}$ ). It is solely parametrized by $\mu, \lambda$, and $\kappa$, and describes signal attenuation as a function of dimensionless frequency $\omega=\frac{2 \pi f L}{v}(f[\mathrm{~Hz}]$ is frequency),

$$
T F=\left|\exp \left[\frac{\left.1-\sqrt{1+4 \mu^{2}(i \omega+\lambda \sqrt{i \omega} \tanh (\kappa \sqrt{i \omega})}\right)}{2 \mu^{2}}\right]\right|^{2} \text {. }
$$

In the related experiments, a 4.5 m-long tube was filled with water vapor using a humidity generator (Fig. 1) that created air of chosen relative humidity $(R H)$. This water vapor was then released as a pulse into a constant, turbulent flow of artificial air (17 lpm, 80\% $\mathrm{N}_{2}, 20 \% \mathrm{O}_{2}$ ), and was transported along a $13.6 \mathrm{~m}$-long tube until it was divided into a $0.5 \mathrm{~m}$-long sampling tube $(7 \mathrm{lpm})$ and a bypass flow. From the sampling tube, the flow was directed into an infrared gas analyzer ${ }^{22}$ and a flow-rate gauge, where after it was joined with the bypass flow in a flow module that recorded the flow rate of the whole setup. All tubes before the infrared analyzer had a diameter of $8 \mathrm{~mm}$ and were made of polytetrafluoroethylene (PTFE). Before the measurements, another $13.6 \mathrm{~m}$-long tube was aged by $20 \mathrm{lpm}$ suction of outdoor air for two weeks near an urban micrometeorologic measurement station ${ }^{23,24}$ so as to provide a realistic reference to a clean tube. The air flow in the measurements was maintained by controlling the pressure of the artificial air and the suction of the flow module. The humidity, temperature, and pressure of the sampled air were logged at $20 \mathrm{~Hz}$, whereas its flow was logged at $0.2 \mathrm{~Hz}$.

So as to verify the proposed mechanism, condensation of drops of water at the tube wall was monitored in a transparent section of the tube (in the "main tube" of Fig. 1) using high-speed optical imaging. A $1 \mathrm{~mm} \times 1 \mathrm{~mm}$ area $(1024 \times$ 1280 pixels) of the (clean and aged) tube wall was videoed at $1 \mathrm{kHz}$ frame rate while a steep change in $R H$, from $42 \%$ to $95 \%$, took place. $R H$ of the air was measured by the same infrared analyzer as before.

High-speed imaging revealed that drops of water were indeed condensed at the tube wall when the $R H$ was increased. In a clean tube these drops were symmetric, had a narrow size distribution, and a uniform spatial distribution at the wall (Fig. 2(a)). In an aged tube, drops had a widely varying size and shape, and a patchy spatial distribution (Fig. 2(b)). Furthermore, condensation of drops in an aged tube started at a lower value of $R H$, and more water was condensed at the wall (Fig. 2).

The peaks of the breakthrough curves for a clean tube in the laboratory experiment were fairly symmetric for all $R H \mathrm{~s}$. A symmetric peak is caused by the symmetric spreading by molecular diffusion and Taylor dispersion of the tracer, while condensation with subsequent evaporation leads to asymmetry of the peak so that there is more weight at later

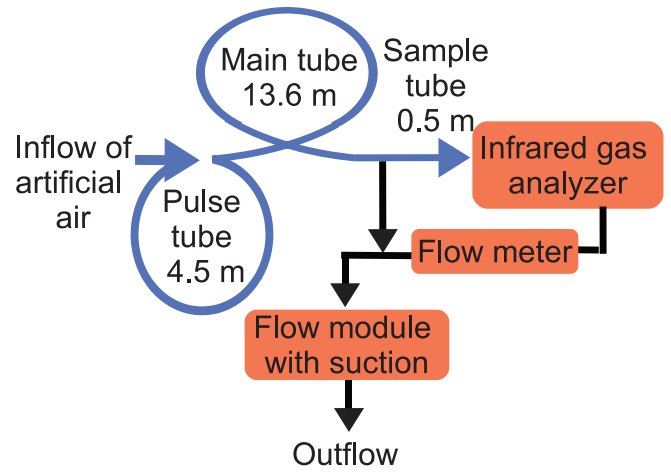

FIG. 1. A schematic layout of the measurement. The blue tubes are made of PTFE. Note that the sizes of different parts of the layout are not in their real relative scales. 


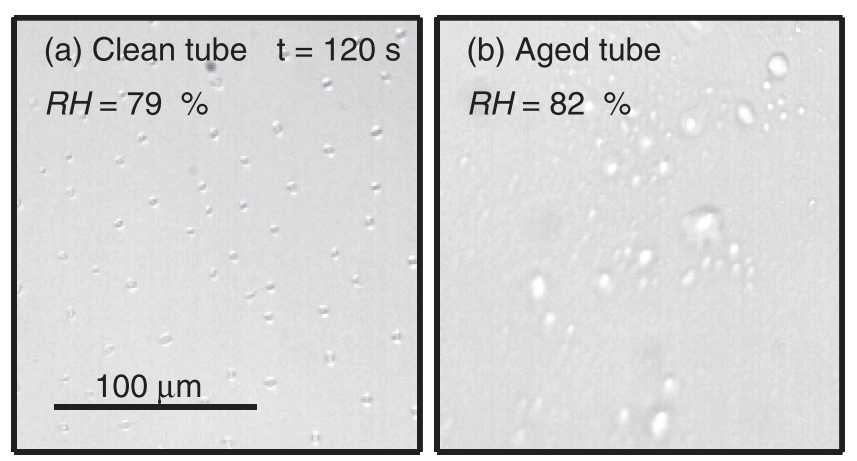

FIG. 2. Condensation of drops of water at tube wall. Images of a clean (a) and an aged tube (b) 2 min after a step change in $R H$ took place (enhanced online) [URL: http://dx.doi.org/10.1063/1.4804639.1].

times. The latter effect was not very prominent in the clean tube, although breakthrough curves were qualitatively similar to those in the aged tube (cf., Fig. 3). Spreading of the pulse by molecular diffusion leads to a pulse that begins to arrive earlier than the "advection time" $(\tau=1)$. Except for the late-time tails, the shapes of the breakthrough curves were mostly explained by Taylor dispersion using a reasonable value for $\mu, \mu=0.035$, by which the early part of the breakthrough curve was explained quite well. Doubling the $\mu$, improved the fit to the late-time behavior, but then agreement with the early-time behavior was lost. It is evident that molecular diffusion and Taylor dispersion do not explain the observed shape of the breakthrough curves for a clean tube and low relative humidities (data not shown). The breakthrough curves for an aged tube were somewhat delayed and had a bit gentler rise of the leading edge (with respect to the $\mu=0.035$-Taylor-dispersion curve), although these features were only minor for the given $R H$ of $32 \%$ (Fig. 3). The main effect of sorption was the strong late-time tailing of the curve, and this was a feature not captured at all by molecular diffusion and Taylor dispersion. Doubling of $\mu$ improved also here the fit to tailing, without being satisfactory, however, but then agreement with the leading edge was completely lost (Fig. 3).

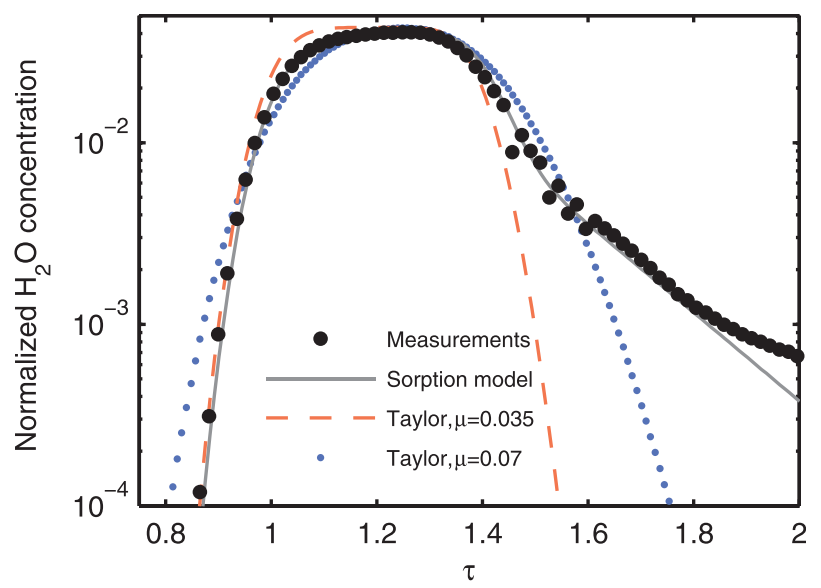

FIG. 3. Breakthrough curve of a pulse of water vapor advected along an aged PTFE tube: Its normalized concentration as a function of dimensionless time $(\tau)$. Shown are the measured data (black dots), their fit by the sorption model (thick gray line), and by Taylor dispersion alone for $\mu=0.035$ (red dashed line) and $\mu=0.07$ (blue small dots). The fit by the sorption model was made with $\lambda=0.09$ and $\kappa=0.62$; the peak $R H$ was $32 \%$ in the experiment.
The sorption model introduced here described the shape of the breakthrough curve also in the case of an aged tube and high $R H$ (data not shown). The steepness of its leading edge was mainly governed by $\lambda$, together with some influence by $\mu$. In a clean tube, $\lambda$ and $\kappa$ were found to be tube properties as they did not depend on $R H$ (Fig. 4). Aging of the tube induces chemical heterogeneity as a result of contamination, and then ( $\lambda$ and) $\lambda \cdot \kappa$ were found to increase for increasing $R H$ (Fig. 4), and the rise of the leading edge of the breakthrough curve became steeper. The slope of the tail-off was governed by $\kappa$ that, for an aged tube, decreased for increasing $R H$ (data not shown). Because of this, the shoulder on the late-time side of the main peak began eventually to dominate the behavior around the main peak, exactly as the finite depth of porous matrix affects the behaviour of the breakthrough curve in matrix diffusion. ${ }^{20}$

There was also a further late-time tail in the breakthrough curves for aged as well as clean tubes, not explained by condensation and subsequent evaporation of water at the tube wall. This tail was caused by (capillary) condensation of water in corners and narrow passages in valves, where evaporation was slowed by stronger adhesion to solid material. ${ }^{26-28}$ Modeling of this effect was not attempted.

Field measurements of turbulent fluctuations of water vapor were also used to verify the performance of the model in frequency space. These data were measured with the eddy-covariance technique, the standard means to measure turbulent vertical fluxes. ${ }^{25}$ Outdoor air was sampled at $31 \mathrm{~m}$ above the ground, and continuously drawn through a $41 \mathrm{~m}$-long tube to a similar infrared gas analyzer as in the laboratory measurements. The flow rate and logging frequency were also kept the same, but the tube material was stainless steel, and a PTFE membrane filter was used in front of the analyzer. In this study, $30 \mathrm{~min}$ power spectra from July 2007 and 2011 in addition to $R H$ measurements of ambient air were used. The water-vapor power spectra were used in combination with power spectra of the airtemperature fluctuations to determine the $T F$ of the measurement system. Temperature fluctuations could be assumed to be unattenuated because measurements were done using a sonic anemometer up in the tower. Scalar similarity between temperature and water-vapor transport was assumed, and the $T F$ was determined as the ratio of the water-vapor power spectrum to that of temperature. More information on the measurements can be found elsewhere. ${ }^{5}$

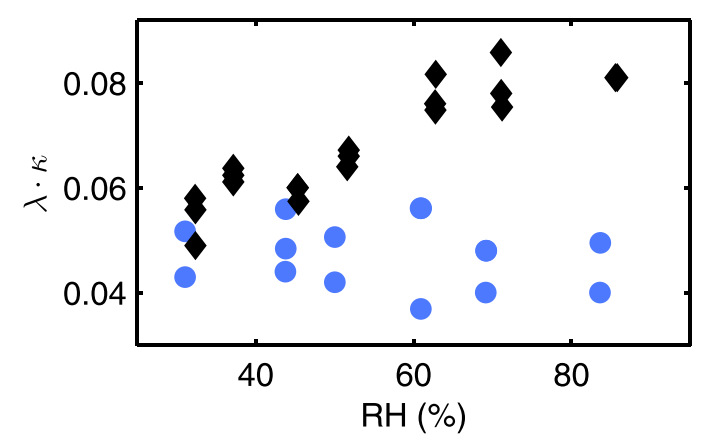

FIG. 4. Product of the fitting variables of the sorption model, $\lambda \cdot \kappa$, as a function of relative humidity $(R H[\%])$ for a clean (light blue spheres) and an aged (black diamonds) tube. 


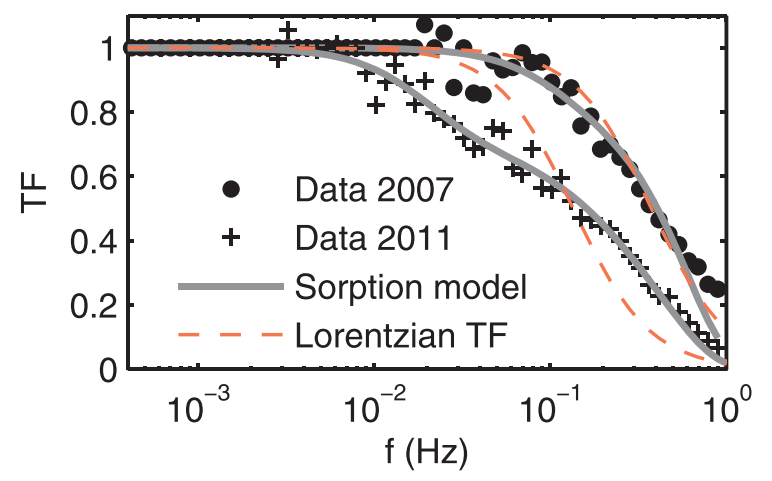

FIG. 5. Transfer functions $(T F)$ as a function of frequency $(f,[\mathrm{~Hz}])$ : Data from field measurements of turbulent fluctuations in water-vapor concentration in 2007 (black dots) and 2011 (black crosses), fits based on the proposed $T F$ (Eq. (4)), and fits based on a Lorentzian TF. $R H=58 \%$ and $\mu=0.024$ for both years. Fitting was made with $\lambda=0.045$ and $\kappa=0.64$ for 2007 , and $\lambda=0.16$ and $\kappa=1.53$ for 2011 .

The TFs of the field measurements show that turbulent water-vapor fluctuations were strongly attenuated in the tube, and more so for an aged tube (Fig. 5). Tube attenuation is often considered as a first-order recursive filter, analogous to a resistor-inductor circuit, which causes a Lorentzian $T F{ }^{3,29}$ The Lorentzian form of $T F$ worked quite well for passive tracers and for a new clean tube, but it was not complex enough to describe sorption processes in an aged tube (Fig. 5 ). In contrast with this, the $T F$ of the proposed model (Eq. (4)) gave excellent fits ( $\lambda$ and $\kappa$ as fitting parameters) to the observed data, it described in particular the "slump" in the interval $10^{-2}-10^{-1} \mathrm{~Hz}$ in the $T F$ of 2011. This kind of "slump" is only observed for a certain range of $\lambda$ and $\kappa$ values of the model, and it is related to the shoulder on the latetime side of the peak in the pulse of Fig. 3. Such a "slump" has been observed before in field measurements, but it has been ignored ${ }^{4}$ and has thus lead to incorrect data correction. The applicability of the sorption model to the $T F$ of field measurement adds to the confidence of the validity of the model.

The fitting variables, $\lambda$ and $\kappa$, depend in principle on the momentary concentration of the water vapor, but then Eq. (2) would be badly nonlinear and would not allow for an analytical solution. For simplicity, we have thus assumed that this dependence can be replaced by that on concentration average, i.e., $\lambda=\lambda\left(C_{\text {ave }}\right)$ and $\kappa=\kappa\left(C_{\text {ave }}\right)$ with a suitably long averaging time $\left(C_{\text {ave }}=\langle C\rangle_{t}\right)$. For clean tubes, this assumption works beautifully as $\lambda$ and $\kappa$ were observed not to depend on the average concentration (e.g., at homogeneously hydrophobic tube walls $\phi$ is not expected to depend on $R H$, at least not for very high values of RH). In aged tubes, parts of the tube wall are covered by hydrophilic contaminants, and $\phi$ can be expected to increase with increasing $R H$ as indeed it was observed to do. Even in this case, the model was found to work well in laboratory as well as field measurements when $\lambda$ and $\kappa$ were used as fitting parameters. Their present values cannot, however, be applied in other situations. On the other hand, their values can be determined by one measurement (breakthrough curve or $T F$ ), and they can then be used until the situation changes appreciably, and their validity can easily be checked by a new fit.
In conclusion, a sorption model for active tracers was introduced, and tested by laboratory and field measurements. The model assumes that an active tracer is transported by a carrier fluid in a tube, it is first (partly) condensed at the tube wall and then evaporated back to the flow, which causes a delay and attenuation in the measured concentration signal. Evaporation of drops was treated as a diffusion-type process. The formation of "drops" of water was verified by highspeed imaging. The behavior of the breakthrough curve of water vapor was well explained by the model, and the model captured in particular the late-time tail in a clean as well as aged tube and for varying humidity, which the traditional Taylor dispersion failed to explain. The three dimensionless variables, which control the behavior of the system, were independent of $R H$ in a clean tube, and behaved in a consistent fashion when $R H$ varied in an aged tube. Furthermore, a $T F$ based on the sorption model explained field measurements: A "slump" in a certain frequency range of $T F$ was captured by the proposed physically based model, while a traditional (Lorentzian) $T F$ failed to describe it. The $T F$ introduced here is applicable to field measurements hampered by signal attenuation.

A.N. is grateful to the Academy of Finland (Projects 1118615 and 263149), the EU (Projects 211574 and 244122), and ERC (Project 227915) for financial support. This study was part of ICOS-EU, ICOS-SA (263149), InGOS, GHG Europe, and DEFROST-projects. We also thank P. Keronen, P. Aalto, and L. Järvi for their help when doing the field measurements.

${ }^{1}$ M. Aubinet, A. Grelle, A. Ibrom, Ü. Rannik, J. Moncrieff, T. Foken, A. S. Kowalski, P. H. Martin, P. Berbigier, C. Bernhofer, R. Clement, J. Elbers, A. Granier, T. Grunwald, K. Morgenstern, K. Pilegaard, C. Rebmann, W. Snijders, R. Valentini, and T. Vesala, Adv. Ecol. Res. 30, 113 (1999).

${ }^{2}$ T. Markkanen, Ü. Rannik, P. Keronen, T. Suni, and T. Vesala, Boreal Environ. Res. 6(1), 65 (2001).

${ }^{3}$ A. Ibrom, E. Dellwik, H. Flyvbjerg, N. O. Jensen, and K. Pilegaard, Agric. Forest Meteorol. 147, 140 (2007).

${ }^{4}$ B. R. K. Runkle, C. Wille, M. Gažovic, and L. Kutzbach, Boundary-Layer Meteorol. 142(3), 401 (2012).

${ }^{5}$ A. Nordbo, L. Järvi, and T. Vesala, Tellus Ser. B 64, 18184 (2012).

${ }^{6}$ G. Taylor, Proc. R. Soc. London Ser. A-Math. Phys. Sci. 219, 186 (1953).

${ }^{7}$ J. Philip, Aust. J. Phys. 16, 454 (1963).

${ }^{8}$ J. Philip, Aust. J. Phys. 16, 287 (1963).

${ }^{9}$ R. Leuning and J. Moncrieff, Boundary-Layer Meteorol. 53, 63 (1990).

${ }^{10}$ G. Taylor, Proc. R. Soc. London Ser. A-Math. Phys. Sci. 223, 446 (1954).

${ }^{11}$ D. H. Lenschow and M. R. Raupach, J. Geophys. Res.-Atmos. 96, 15259 (1991).

${ }^{12}$ W. J. Massman, J. Geophys. Res.-Atmos. 96, 15269 (1991).

${ }^{13}$ W. J. Massman and A. Ibrom, Atmos. Chem. Phys. 8, 6245 (2008).

${ }^{14}$ A. Nordbo and G. G. Katul, Boundary-Layer Meteorol. 146(1), 81 (2013).

${ }^{15}$ T. A. H. Nguyen, A. V. Nguyen, M. A. Hampton, Z. P. Xu, L. Huang, and V. Rudolph, Chem. Eng. Sci. 69, 522 (2012).

${ }^{16}$ E. Sultan, A. Boudaoud, and M. B. Amar, J. Fluid Mech. 543, 183 (2005).

${ }^{17}$ W. Brutsaert, J. Geophys. Res. 70, 5017, doi:10.1029/JZ070i020p05017 (1965).

${ }^{18}$ M. Sahimi, Flow and Transport in Porous Media and Fractured Rock (VCH, Weinheim, Germany, 1995), p. 718.

${ }^{19}$ See supplementary material at http://dx.doi.org/10.1063/1.4804639 for a solution for Eqs. (2) and (4) and for Taylor dispersion in a pipe flow.

${ }^{20}$ P. Kekäläinen, M. Voutilainen, A. Poteri, P. Höltta, A. Hautojärvi, and J. Timonen, Transp. Porous Media 87, 125 (2011).

${ }^{21}$ I. Neretnieks, J. Geophys. Res. 85(B8), 4379, doi:10.1029/JB085iB08p04379 (1980). 
${ }^{22}$ G. G. Burba, D. K. Mcdermitt, D. J. Anderson, M. D. Furtaw, and R. D. Eckles, Tellus Ser. B 62, 743 (2010).

${ }^{23}$ L. Järvi, H. Hannuniemi, T. Hussein, H. Junninen, P. P. Aalto, R. Hillamo, T. Mäkelä, P. Keronen, E. Siivola, T. Vesala, and M. Kulmala, Boreal Environ. Res. 14, 86 (2009).

${ }^{24}$ A. Nordbo, L. Järvi, S. Haapanala, J. Moilanen, and T. Vesala, BoundaryLayer Meteorol. 146, 469 (2013).
${ }^{25}$ M. Aubinet, T. Vesala, and D. Papale, Eddy Covariance-A Practical Guide to Measurement and Data Analysis (Springer Atmospheric Sciences, The Netherlands, 2012), p. 438.

${ }^{26} \mathrm{~W}$. Thomson, Proc. R. Soc. Edinburgh 7, 63 (1870).

${ }^{27}$ W. Thomson, Philos. Mag. 42, 448 (1871).

${ }^{28}$ L. R. Fisher and J. N. Israelachvili, Nature 277, 548 (1979).

${ }^{29}$ W. Eugster and W. Senn, Boundary-Layer Meteorol. 74, 321 (1995). 